\title{
Paraganglioma de cauda equina
}

\section{Paraganglioma of the cauda equina}

\author{
M. Gelabert-González, E. Aran-Echabe y R. Serramito-García
}

\section{Sr. Director:}

Hemos leído con interés el trabajo publicado por Undabeitia-Huertas y $\mathrm{col}^{1}$ : $\mathrm{Pa}$ raganglioma del filum terminal como causa de síndrome de cauda equina, y nos gustaría hacer algún comentario al mismo en base a nuestra propia experiencia con este tipo de tumores ${ }^{2}$.

Los paragangliomas son tumores neuroendocrinos del sistema paraganglionar extra-adrenal, que tiene su origen en las células neuroendocrinas de la cresta neural. La gran mayoría se localizan en la región del glomus yugular y cuerpo carotídeo y dentro del sistema nervioso central se han descrito casos localizados en: glándula pineal e hipófisis, ángulo ponto-cerebeloso así como en la cauda equina y filum terminal.

Los autores dan a entender en su trabajo que se originan en exclusividad en el filum terminal y solo ocasionalmente en las raíces nerviosas. En nuestra revisión de la literatura publicada en el año 2005, y sobre un análisis de 174 casos, observamos que el $33,9 \%$ de los paragangliomas lumbares
(57 casos) se originaban (están adheridos) a una raíz nerviosa, el $28,1 \%$ en el filum terminal (52 casos) y $<3 \%$ en el cono medular. En el resto de casos no se especificaba con claridad el punto donde el tumor estaba adherido $^{2}$.

Con relación a la sintomatología, los autores indican que no se han publicado casos relacionados con crisis catecolaminérgicas. Uno de los casos publicados que acompañan a la revisión del año 2005, una paciente de 62 años con un paraganglioma a nivel L3-L4, presentó al iniciar la manipulación del tumor, un rápido incremento de la tensión arterial acompañado de taquicardia. Al parar con la manipulación del tumor, se normalizaron las cifras de tensión arterial y la frecuencia cardíaca, repitiéndose la misma situación con nuevas manipulaciones. Tras ligadura del pedículo vascular se pudo continuar con la extirpación completa del tumor sin que produjesen nuevos eventos hipertensivos. En la revisión de la literatura existen al menos otros tres casos similares ${ }^{3,4}$ y en el paciente descrito por Böker y col$^{5}$ se demostraron niveles altos de catecolaminas en orina.
Servicio de Neurocirugía. Hospital Clínico Universitario de Santiago de Compostela. Departamento de Cirugía. Universidad de Santiago de Compostela.

Recepción: 27 de noviembre de 2013

Aceptación provisional: 5 de diciembre de 2013

Aceptación definitiva: 18 de diciembre de 2013

\author{
Correspondencia: \\ Miguel Gelabert-González \\ Departamento de Cirugía \\ Universidad de Santiago de Compostela \\ San Francisco, 1 \\ 15705 Santiago de Compostela \\ E-mail: miguel.gelabert@usc.es
}


Compartimos con los autores la opinión de que el diagnóstico preoperatorio es difícil, ya que estos tumores representan menos del $2 \%$ de todos los tumores medulares $^{6}$ y habitualmente se confunden preoperatoriamente con schwannomas y meningiomas. Sin embargo, se han descrito en la resonancia magnética con contraste la presencia de lesiones serpiginosas que corresponden a los vasos del tumor?

\section{BIBLIOGRAFÍA}

1. Undabeitia-Huertas J, Noboa R, Jové R, Boix M, Gatius S, Nogues P. Paraganglioma del filum terminal como causa de síndrome de cauda equina. An Sist Sanit Navar 2013; 36: 347351.
2. Gelabert-González M. Paragangliomas of the lumbar region. Report of two cases and review of the literature. J Neurosurg Spine 2005; 2: 354-365.

3. Toyota B, Barr HW, Ramsay D. Hemodynamic activity associated with a paraganglioma of the cauda equine. Case report. J Neurosurg 1993; 79: 451-455.

4. González-Campora R, López J, Castellanos $\mathrm{F}$ et al. Paraganglioma of de la cauda equina. Rev Neurol 1986; 14 217-224.

5. Böker DK, Wassmann H, Solymosi L. Paragangliomas of the spinal canal. Surg Neurol 1983; 19: 461-468.

6. Gelabert-González M. Tumores medulares primarios. Análisis de una serie de 168 pacientes. Rev Neurol 2007; 44: 269-274.

7. Lamer S, Carlier RY, Parker F, Lacroix C, Larue F, DE LATour B et al. Paraganglioma of the cauda equine. MR findings. One case. J Neuroradiol 1997; 24: 215-217. 